\title{
Editorial Editorial
}

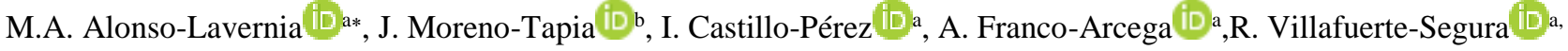 \\ a Área Académica de Computación y Electrónica, Universidad Autónoma del Estado de Hidalgo, 42184, Pachuca, Hidalgo, México. \\ b Área Académica de Ciencias de la Educación. Universidad Autónoma del Estado de Hidalgo, 42184, Pachuca, Hidalgo, México.
}

\section{Apreciables lectores de Pädi}

Al conjuntar las tendencias actuales y futuras en el sector educativo con la situación de adversidad vivida en los últimos meses debido a la pandemia mundial del Coronavirus, se hace aún más imperante la búsqueda de alternativas basadas en tecnologías de vanguardia para poder lograr la excelencia en los procesos de enseñanza y aprendizaje, llevando la educación a niveles que satisfagan características como: la independencia del tiempo y del espacio, la adaptación a los diferentes individuos y contextos, la diversidad en la forma de expresar los saberes, pero sobre todo, la búsqueda de motivación de los aprendices que favorezca el desarrollo de capacidades de autonomía, gestión y competitividad para el logro de sus retos personales y por consiguiente, el beneficio del lugar donde se inserte y de la sociedad en general.

Este número especial tiene el propósito de compartir trabajos enfocados a dar respuesta a problemáticas específicas presentadas en diversas áreas del conocimiento y contextos del quehacer educativo, favoreciendo al estudiante en su proceso de formación y a la vez, al docente en su labor de instrucción. De ahí, que las aportaciones presentadas proceden de autores adscritos a diversas instituciones educativas nacionales e internacionales, siendo éstas:

- Humboldt International University, USA

- Universidad de Cienfuegos Carlos Rafael Rodríguez, Cuba

- Universidad Autónoma de Madrid, España

- Universidad Veracruzana, México

- Instituto Tecnológico de Sonora, México

- Instituto Politécnico Nacional, México

- Benemérita Universidad Autónoma de Puebla, México

- Universidad de Guadalajara, México

- Universidad Tecnológica de Tulancingo, México

- Instituto Tecnológico Superior del Oriente del Estado de Hidalgo, México

- Universidad Autónoma del Estado de México, México

- Universidad Autónoma del Estado de Hidalgo, México.

De igual manera, en este contexto, se manifiesta la colaboración entre universidades a través de aportaciones que presentan los resultados del trabajo conjunto de sus autores, lo cual impacta favorablemente en la obtención de mayores y mejores resultados en la investigación y en los índices de citación. Las colaboraciones científicas identificadas en este número son:

- Humboldt International University - Universidad Autónoma del Estado de Hidalgo

- Universidad Veracruzana - Instituto Tecnológico de Sonora - Benemérita Universidad Autónoma de Puebla

- Universidad Veracruzana - Universidad Autónoma de Madrid

- Universidad Autónoma del Estado de Hidalgo Universidad de Guadalajara

- Universidad Autónoma del Estado de Hidalgo Universidad Autónoma del Estado de México

Respecto al contenido de las aportaciones y mirando el contexto del uso de las Tecnologías Avanzadas en la Educación desde perspectivas diferentes, se han organizado los trabajos de este número en tres secciones que definen áreas de oportunidad para engrandecer la labor educativa y las cuales son:

- Modelos y estrategias pedagógicas basadas en la tecnología

- Herramientas y aplicaciones para innovar en la educación

- Tecnologías inteligentes para la educación

Antes de brindar una breve descripción de los trabajos publicados en esta emisión especial del Pädi Boletín Científico de Ciencias Básicas e Ingenierías, el equipo editorial quiere expresar su más afectuoso reconocimiento a todos los autores que han contribuido con sus trabajos y apoyado en la labor de revisión de los mismos.

\section{Modelos y estrategias pedagógicas basadas en la tecnología}

Esta primera sección presenta siete artículos de revisión y experiencias investigativas centradas en modelos y estrategias pedagógicas que utilizan como mediación a la tecnología. El lector encontrará referentes prácticos importantes que pueden ser útiles como modelos a seguir para innovar en sus propios contextos. Cada uno de los artículos que componen esta sección se centran en aspectos diferentes que son complementarios entre si desde una visión integrada que presenta elementos y referentes importantes a considerar por parte de cualquier comunidad educativa que tenga el interés de innovar en el diseño pedagógico

\footnotetext{
*Autor para la correspondencia: marial@uaeh.edu.mx Pérez), afranco@uaeh.edu.mx (Anilu Franco-Arcega), villafuerte@uaeh.edu.mx (Raúl Villafuerte-Segura).

Correo electrónico: marial@ uaeh.edu.mx (María de los Ángeles Alonso-Lavernia), javier_moreno@uaeh.edu.mx (Javier Moreno-Tapia), ilianac@uaeh.edu.mx (Iliana Castillo- 
de sus programas educativos a través de la mediación de la tecnología.

El manuscrito titulado "Comunidad de aprendizaje y gestión del conocimiento en universidades" describe la relación entre estos conceptos en el contexto de un grupo de profesores investigadores pertenecientes a centros de investigación de una universidad pública. Se resalta la importancia de promover comunidades de aprendizaje y que el propósito de las mismas este estratégicamente regulado con la intención de gestionar conocimiento para asegurar la calidad y vigencia de la comunidad creada. Todo ello, a través de la mediación de herramientas Web 2.0 en la interacción de los miembros (Martínez-Lazcano et al., 2021).

Por su parte, el manuscrito denominado "Resolución de problemas en el aprendizaje y enseñanza de las matemáticas" permite identificar los aspectos de una comunidad de aprendizaje que contribuyen a la comprensión de problemas matemáticos a partir de la aportación de autores matemáticos que son un referente fundamental en la concreción de una aproximación didáctica (Barrera-Mora et al., 2021).

Mientras que en "Taller de Diseño Editorial bajo una Estrategia Didáctica basada en las Tecnologías de la Información y la Comunicación" considera a estas últimas como instrumentos que han promovido cambios en los paradigmas educativos y que gestionan la promoción de estrategias didácticas basados en metodologías activas donde el rol del aprendiz y docente se ajustan; los primeros, personas autónomas y activos en su proceso de aprendizaje y los segundos, como promotores y guías que diseñan y ajustan ayudas pedagógicas aprovechando la medicación de las tecnologías. Se describe un ejemplo concreto de estos roles y uso de estrategias en una asignatura de la Licenciatura en Diseño Gráfico (Gaona-Franco et al., 2021).

En "Estrategia didáctica para la enseñanza matemática en turismo" se centra en la descripción de una reflexión sobre las prácticas didácticas de la temática mencionada en el contexto de la pandemia por COVID-19, la cual se centra en la teoría antropológica didáctica que permite recuperar las experiencias que visibilizan las dificultades que enfrentan los docentes y estudiantes en el contexto referido. Concluye que se tienen dificultades para interpretar el lenguaje formal al igual que en la solución de la situación matemática y el uso de la tecnología, que dependen de las asesorías que ofrece el docente (Briones-Juárez et al., 2021).

El manuscrito titulado "Enseñanza remota de emergencia durante la pandemia de Coronavirus" evalúa el uso de una estrategia didáctica en el contexto de este entorno emergente a través del modelo de evaluación CIPP de Stufflebeam para evaluar el contexto y el insumo de la asignatura de Estadística Descriptiva en una universidad pública. Los resultados revelan que se obtuvo la cuantificación de dedicación a sus estudios, el tiempo disponible de uso de cómputo para las sesiones. Además, se valoro el estado emocional presente resultado de la contingencia sanitaria (Ochoa-Alcántar et al., 2021).

Continuando con "Educación a distancia: De la Prehistoria a las Pandemias Modernas, una Revisión Breve y La situación actual del nivel Medio Superior en México" se centra en el análisis del ser humano quien tiene una gama de necesidades en su papel dentro de un entorno social. Desde esta perspectiva el propósito puntual del documento es integrar una síntesis desde los inicios de la educación a distancia hasta el escenario emergente que implica la contingencia sanitaria en el contexto de la educación media superior en México (Arellano-Calderón et al., 2021).

El último manuscrito de esta primera sección se titula "Marcos de Competencias Digitales relacionados con Seguridad para docentes" y tiene como propósito realizar una revisión bibliográfica acerca de las competencias digitales relacionadas con aspectos de seguridad digital. El análisis de contenido permitió identificar siete marcos que proponen prácticas seguras, legales y éticas cuando los estudiantes usan herramientas digitales. Se concluye estableciendo la necesidad de fortalecer la educación en conocimientos, hábitos y estrategias prácticas para asegurar la seguridad digital (Amador-Alarcón et al., 2021).

\section{Herramientas y aplicaciones para innovar en la educación}

Esta segunda sección está enfocada a trabajos que se refieren a la evolución tecnológica y cómo ésta ha originado grandes cambios en la diversificación del acceso a la información, la cual ha impactado directamente a la educación, ocasionando la innovación en el desarrollo de sistemas, aplicaciones y herramientas que impulsan el acceso al conocimiento y el desarrollo de innumerables habilidades, además de ofrecer una guía en la obtención de indicadores para la estimulación del neurodesarrollo desde edades muy tempranas, instrumentos que permiten diagnosticar el conocimiento y uso de las tecnologías en futuros docentes, así como hacer explícitas las necesidades de instrumentos que apoyen ciertas áreas de la evaluación del aprendizaje en modalidad virtual.

En el manuscrito titulado "Sistema de enseñanza para la técnica de agrupamiento k-means" se presenta una herramienta de apoyo a la enseñanza que permite descubrir paso a paso, cómo se construye la partición de un conjunto de datos en la aplicación del algoritmo k-means, el cual es la técnica de clasificación no supervisada más utilizada en el procesamiento de datos aplicado a entornos reales y cotidianos (Franco-Arcega, A., et al., 2021).

En el manuscrito "Propuesta para la gamificación de experimentos en los laboratorios virtuales" se describe el uso de la Metodología para el Desarrollo de Entornos Educativos de Realidad Virtual (MEDEERV) para el desarrollo de un prototipo en un motor de videojuegos que utiliza técnicas de modelado de realidad virtual para presentar el tema de caída libre de los cuerpos con aceleración constante, además de presentar los resultados de una evaluación heurística del grado de usabilidad de dicho prototipo (Torres-Samperio, G. A., et al., 2021).

Mientras que en "Revisión de la literatura digital de instrumentos evaluativos para modalidad virtual de aprendizaje" se exponen los resultados de un estudio bajo la metodología de análisis documental, desde un enfoque cuantitativo, de una selección de textos científicos indexados que versan sobre instrumentos de evaluación en la modalidad virtual de aprendizaje (Gómez-Acosta, E. I., et al., 2021).

En "Guía de observación para la exploración de las inteligencias múltiples en los niños de cinco a seis años de edad" se ofrece un instructivo con estándares y explicaciones para la aplicación de una serie de indicadores, posibilitando a los educadores conocer las particularidades individuales de sus educandos y rediseñar las estrategias de intervención educativa que estimulen su neurodesarrollo (Alfonso-Amaro, Y., et al., 2021).

Por otro lado, una herramienta CASE que permite a los estudiantes del área de computación reafirmar conceptos teóricos de la rama del desarrollo web a través del microaprendizaje, así como aplicar los conocimientos necesarios para el desarrollo de aplicaciones de tipo SPA (Single Page Application) que implementen una arquitectura de tipo MVC (Modelo-VistaControlador), es presentada en "C-ToolP: Herramienta de apoyo para la enseñanza de desarrollo Web-SPA-MVC" (García-Islas, L. H., et al., 2021). 
Finalmente, en esta sección se presenta "Instrumento de autopercepción de competencia digital para futuros docentes" donde se describe la creación, validación y fiabilidad de un instrumento ad-hoc con 40 ítems distribuidos en áreas del conocimiento como la pedagogía, conocimientos técnicos, aspectos sociales, éticos y legales, además del uso de las TIC, cuyos resultados facilitan el diagnóstico de los estudiantes respecto al conocimiento y uso de las tecnologías, a nivel instrumental y pedagógico como parte de su formación profesional docente (Cañete-Estigarribia, D. L., et al., 2021).

\section{Tecnologías inteligentes para la educación}

La evolución de los procesos de enseñanza y aprendizaje requieren que nuevas tendencias y herramientas sean empleadas por los facilitadores para la mejora de dichos procesos. Disciplinas como la Inteligencia Computacional ofrecen diversas técnicas, métodos y estrategias que pueden ser aplicadas con el fin de encontrar y proponer novedosas soluciones que personalicen la enseñanza, identifiquen problemáticas específicas de los alumnos, provean de estrategias didácticas para reafirmar el conocimiento, entre otros aspectos.

En esta tercera sección, se presentan cinco manuscritos relacionados a tecnologías inteligentes aplicadas para la mejora de diferentes enfoques educativos.

El manuscrito denominado "Simulación de un sistema de péndulos con GNU Octave" introduce una herramienta didáctica que simula sistemas mecánicos que emplean algunas ecuaciones diferenciales, presentando el caso particular de un sistema de péndulos. Para llevar a cabo estas simulaciones, el trabajo utiliza superposición de imágenes que modelan la dinámica correspondiente del sistema (Ávila-Pozos, R., et al., 2021).

Por su parte, el manuscrito "Nano transistores de efecto de campo tipo aleta para aplicaciones digitales" presenta un panorama de la construcción y funcionamiento de transistores de efecto de campo de aleta a través de diagramas esquemáticos. Su objetivo es permitir a los alumnos identificar y conocer las características del tipo de compuertas que son la base para el diseño y construcción de la mayoría de los dispositivos digitales utilizados en los aparatos electrónicos (González-Vidal, J.L., et al., 2021).

En "Detección de estudiantes que copian en el aula usando Redes Neuronales Convolucionales" se muestran los resultados del uso de algoritmos inteligentes para la identificación de alumnos que copian en la aplicación de exámenes presenciales en las aulas, a través del procesamiento de imágenes (Cruz-Guerrero, $R$., et al., 2021).

Continuando con "Mecatrónica educativa: soporte de la enseñanza-aprendizaje de educación básica en Hidalgo" donde se propone el desarrollo de prototipos y material didáctico con un enfoque novedoso, basados en mecatrónica educativa, para fomentar el interés científico, tecnológico y de innovación de estudiantes de educación básica del Estado de Hidalgo (GonzálezIslas, J.C., et al., 2021).

Finalmente, este número presenta el manuscrito "Maqueta interactiva I4.0 como medio para la enseñanza-aprendizaje del uso de sistemas ciber-físicos para la Industria 4.0" en donde se describe el desarrollo de un sistema ciber-físico, a través de una maqueta y una serie de prácticas, que permiten generar y reforzar en los estudiantes las capacidades requeridas por la Industria 4.0, tales como el uso de computación en la nube, internet de las cosas y recorridos virtuales (Cuaya-Simbro, G., et al., 2021).

\section{Referencias}

Alfonso-Amaro, Y., Carreño-Ortega, D., \& Massani-Enríquez, J. F. (2021) Guía de observación para la exploración de las inteligencias múltiples en los niños de cinco a seis años de edad. Pädi Boletín Científico de Ciencias Básicas e Ingenierías del ICBI, 9(Especial), 74-78. Recuperado de https://doi.org/10.29057/icbi.v9iEspecial.7258

Amador-Alarcón, M. P., Torres-Gastelú, C. A., Lagunes-Domínguez, A., Angulo-Armenta, J., Medina-Cruz, H. \& Argüello-Rosales, C.A. (2021). Pädi Boletín Científico de Ciencias Básicas e Ingenierías del ICBI, 9(Especial), 48-52. Recuperado https://doi.org/10.29057/icbi.v9iEspecial.7490

Arellano-Calderón, F., Cano-Rosas, J. L., González-Baldovinos, D. L. \& Mendiola-Medellín, M. E. (2021). Educación a distancia: De la Prehistoria a las Pandemias Modernas, una Revisión Breve y La situación actual del nivel Medio Superior en México. Pädi Boletín Científico de Ciencias Básicas e Ingenierías del ICBI, 9(Especial), 42-47. Recuperado de https://doi.org/10.29057/icbi.v9iEspecial.7483

Ávila-Pozos, R., Cruz-Castillo, R., Santillán-Hernández, A.S., TemoltziÁvila, R. (2021). Simulación de un sistema de péndulos con GNU Octave. Pädi Boletín Científico de Ciencias Básicas e Ingenierías del ICBI, 9(Especial), 94-98. Recuperado de https://doi.org/10.29057/icbi.v9iEspecial.7196

Barrera-Mora, F., Reyes-Rodríguez, A., Campos-Nava, M., \& RodríguezÁlvarez, C. (2021). Resolución de problemas en el aprendizaje y enseñanza de las matemáticas. Pädi Boletín Científico de Ciencias Básicas e Ingenierías del ICBI, 9(Especial), 10-17. Recuperado de https://doi.org/10.29057/icbi.v9iEspecial.7051

Briones-Juárez, A. \& Velásquez-Castro, J.A. (2021). Estrategia didáctica para la enseñanza matemática en turismo. Pädi Boletín Científico de Ciencias Básicas e Ingenierías del ICBI, 9(Especial), 29-35. Recuperado de https://doi.org/10.29057/icbi.v9iEspecial.7212

Cañete-Estigarribia, D. L., Torres-Gastelú, C. A., Lagunes-Domínguez, A., \& Gómez-García, M. (2021). Instrumento de autopercepción de competencia digital para futuros docentes. Pädi Boletín Científico de Ciencias Básicas e Ingenierías del ICBI, 9(Especial), 85-93. Recuperado de https://doi.org/10.29057/icbi.v9iEspecial.7488

Cruz Guerrero, R., \& Gutiérrez Fragoso, K. (2021). Detección de estudiantes que copian en el aula usando Redes Neuronales Convolucionales. Pädi Boletín Científico de Ciencias Básicas e Ingenierías del ICBI, 9(Especial), 106-109. Recuperado https://doi.org/10.29057/icbi.v9iEspecial.7492

Cuaya-Simbro, G., Zaragoza-Ramírez, J. A., Covarrubias-Cruz, U.Y., Martínez-Rodríguez, J., Ruiz- Hernández, E., \& Gutiérrez-Fragoso, K. (2021). Maqueta interactiva I4.0 como medio para la enseñanzaaprendizaje del uso de sistemas ciber-físicos para la Industria 4.0. Pädi Boletín Científico de Ciencias Básicas e Ingenierías del ICBI 9(Especial), 118-127. Recuperado de https://doi.org/10.29057/icbi.v9iEspecial.7193

Franco-Arcega, A., Sobrevilla-Solís, V. I., Gutiérrez-Sánchez, M. J., GarcíaIslas, L. H., Suárez-Navarrete, A., \& Rueda-Soriano, E. (2021). Sistema de enseñanza para la técnica de agrupamiento k-means. Pädi Boletín Científico de Ciencias Básicas e Ingenierías del ICBI, 9(Especial), 5358. Recuperado de https://doi.org/10.29057/icbi.v9iEspecial.7384

Gaona-Franco, M.E., Alonso-Lavernia, M.A., Castillo-Pérez, I. \& MuñozSánchez, Y. (2021). Taller de Diseño Editorial bajo una Estrategia Didáctica basada en las Tecnologías de la Información y la Comunicación. Pädi Boletín Científico de Ciencias Básicas e Ingenierías del ICBI, 9(Especial), 18-28. Recuperado de https://doi.org/10.29057/icbi.v9iEspecial.7475

García-Islas, L. H., Franco-Sánchez, K. D., Samperio-Monroy, T. I., PérezTavera, I. H., \& Ocampo-López, A. (2021). C-ToolP: Herramienta de apoyo para la enseñanza de desarrollo Web-SPA-MVC. Pädi Boletín Científico de Ciencias Básicas e Ingenierías del ICBI, 9(Especial), 7984. Recuperado de https://doi.org/10.29057/icbi.v9iEspecial.7470

Gómez-Acosta, E. I., Lagunes-Domínguez, A., \& Torres-Gastelú, C. A. (2021). Revisión de la literatura digital de instrumentos evaluativos para modalidad virtual de aprendizaje. Pädi Boletín Científico de Ciencias Básicas e Ingenierías del ICBI, 9(Especial), 68-73. Recuperado de https://doi.org/10.29057/icbi.v9iEspecial.7462

González-Islas, J.C., Godínez-Garrido, G., González-Rosas, A., \& OrtegaMarín, B.A. (2021). Mecatrónica educativa: soporte de la enseñanzaaprendizaje de educación básica en Hidalgo. Pädi Boletín Científico de Ciencias Básicas e Ingenierías del ICBI, 9(Especial), 110-117. Recuperado de https://doi.org/10.29057/icbi.v9iEspecial.7197 
González-Vidal, J.L., Veloz-Rodríguez, M.A., Lira-Hernández, I.A., GarcíaRivera, M., Vázquez- García, R.A., \& Raygoza-Panduro, J.J. (2021). Nano transistores de efecto de campo tipo aleta para aplicaciones digitales. Pädi Boletín Científico de Ciencias Básicas e Ingenierías del ICBI, 9(Especial), 99-105. Recuperado de https://doi.org/10.29057/icbi.v9iEspecial.7218

Martínez-Lazcano, V. \& Prieto-Barboza, E. A. (2021). Comunidad de aprendizaje y gestión del conocimiento en universidades. Pädi Boletín Científico de Ciencias Básicas e Ingenierías del ICBI, 9(Especial), 1-9. Recuperado de https://doi.org/10.29057/icbi.v9iEspecial.7102
Ochoa-Alcántar, J. M., García-López, R. I. \& Cuevas-Salazar, O. (2021) Enseñanza remota de emergencia durante la pandemia de Coronavirus. Pädi Boletín Científico de Ciencias Básicas e Ingenierías del ICBI, 9(Especial), 36-41. Recuperado https://doi.org/10.29057/icbi.v9iEspecial.7489

Torres-Samperio, G. A., Gutiérrez-Sánchez, M. J., Sánchez-Espinoza, J., Suárez-Navarrete, A., \& Hernández-Sánchez, D. (2021). Propuesta para la gamificación de experimentos en los laboratorios virtuales. Pädi Boletín Científico de Ciencias Básicas e Ingenierías del ICBI, 9(Especial), 5967. Recuperado de https://doi.org/10.29057/icbi.v9iEspecial.7461 